\title{
Device verification and compatibility for heterogeneous semantic IoT systems
}

\begin{abstract}
Interoperability has always been ambiguous for heterogeneous IoT device management. A lot work done in the past focused on the protocol management and to the extent of application inter-operation. Yet, semantic gap in ensuring federated message exchange among heterogeneous IoT devices remain as the significant challenge. In this paper, we illustrate device compatibility using semantic rule between multiple devices in a heterogeneous IoT system. The proposed approach is SWRL based on the light weight IoT ontologies which was modeled and also correspond to segregation of devices based on device compatibility. SWRL was deployed for verifying the compatibility between multiple devices using the semantic rule engine based on bespoke parameters. Performance evaluation was carried out with execution time of transferring axioms of ontology to rules engine, execution time of rules in the engine and the time taken for axioms reflected to the ontology between all device instances defined in the ontology.
\end{abstract}

Keyword: Compatibility; Ontology; Semantic web rule language; SPARQL; IoT 